\title{
Performance do Edifício: Produtos e Processo para Integração no Projeto Arquitetônico
}

\author{
Building Performance: Products and a Process for Architectural Design Integration
}

\author{
Carlos Alejandro Nome \\ Universidade Federal da Paraíba, Brasil \\ carlos.nome@gmail.com \\ Natália Queiroz \\ Universidade Federal de Pernambuco, Brasil \\ nataliaqueiroz.arq@gmail.com
}

\begin{abstract}
The paper presents a case study on BIM supported design processes and its intersections with discussions about sustainability and energy efficiency simulations. The objective of the study is to identify different levels of detail for BIM models that are compatible with sustainability and energy efficiency simulations. As a result an integrated design, model and simulation process is proposed and validated.
\end{abstract}

Keywords: Simulação; BIM; Projeto integrado; Métodos.

\section{Introdução}

Este artigo apresenta um estudo de caso sobre intersecções entre considerações de sustentabilidade e processos de projeto apoiados em modelos BIM. O objetivo é identificar diferentes estágios de modelagem compatíveis com questões específicas de sustentabilidade.

Com o desenvolvimento de tecnologias de modelagem de informação das edificações, os processos de integração de projetos passam a ter um veiculo que habilita a sua implementação prática (Eastman, 2008). É recomendado que seja considerada uma integração em rede em vez de linear (Boddy, Rezgul, Cooper, \& Wetherill, 2007)

A utilização de processos integrados permite, por meio de tomada de decisões nas fases inicias do projeto, a maximização de impactos positivos e a minimização de custo de mudanças (AIA-CC, 2007). Desta forma, é fundamental que em países como o Brasil sejam implementadas estas tecnologias para a otimização do aproveitamento dos recursos humanos e econômicos disponíveis.

\section{Revisão Bibliográfica}

Os conceitos de sustentabilidade e eficiência energética são as lentes sob a qual este trabalho é observado. Este considera que edifícios são potenciais poluentes e os arquitetos são responsáveis éticos por reduzir seu impacto (Roaf, 2012).
Sustentabilidade, eficiência energética e uso de simuladores de desempenho na arquitetura.

Em 1988, a ONU definiu desenvolvimento sustentável em três vertentes principais: aquilo que é economicamente viável, ecologicamente correto, e socialmente justo (Friedrich-NaumannStiftung., 1988). No Brasil os edifícios consomem cerca de $43 \%$ da energia gerada. A construção civil também é responsável por gerar pouco mais da metade do lixo do país (Brasil, 2002) (Lamberts, 2004) (Bachtold, 2008). É imperativo que a concepção do projeto passe a ser mais criteriosa, considerando o uso racional da água, da energia, dos recursos naturais, a diminuição do impacto no terreno e na produção de resíduos (Roaf, 2012).

Para a adoção de estratégias bioclimáticas e uso eficiente de energia, é fundamental entendimento dos fundamentos físicos relacionados. Não há garantias de que a aplicação exclusiva de técnica científica resulte em boa arquitetura, assim como, não há garantia que somente intuição ou experiência produza edificações eficientes. Por isso, é necessário combinar ciência e sensibilidade (Green building rating system : for new construction and core \& shell projects : based on LEED 2009, reference guide, 2011).

Simuladores de desempenho colaboram com a avaliação térmica e energética de edificações. É possível estimar o consumo de energia e dimensionar o impacto ambiental antes da execução do edifício (Mendes, Westphal, Lamberts, \& Neto, 2005). Venâncio (2012) afirma que simuladores contribuem para o entendimento e 
solução de dilemas surgidos durante o projeto e também na percepção de dilemas desconhecidos pelos projetistas.

Por se tratarem de softwares muitas vezes complexos, que dependem de domínio, sua aplicação por arquitetos é limitada e recente (Mendes et al., 2005) (Venâncio, 2012). Estes tipicamente ocorrem em etapas posteriores ao anteprojeto e são realizados por consultores de forma isolada. Essa falta de integração gera problemas de compatibilidade (Venâncio, 2012). Em resposta a este cenário surgem softwares simplificados visando uso de simulação por arquitetos desde as primeiras fases de projeto.

Venâncio, 2012 propõe uso de simulações simplificadas, intermediárias e complexas dividindo a sua aplicação no processo projetual (esboço, anteprojeto e detalhamento). Segundo o autor, assim é possível integrar melhor condicionantes de conforto ambiental e eficiência energética ao projeto. Venâncio defende o uso de simulação complexa em projetos mais "pragmáticos" ainda em fases preliminares. Já edifícios menos exigentes, podem utilizar-se simulações simplificadas e intermediárias.

\section{Programação arquitetônica, reflexão na ação, projeto baseado em evidências e nível de detalhamento}

O conceito de prática reflexiva está atrelado à reflexão durante o fazer (Schön, 1987). Consiste tanto de práticas de consideração retroativa sobre resultados obtidos, quanto de prática de consideração propositiva no ato da concepção (Schön, 1987). Em termos metodológicos, implica em incluir no processo de projeto momentos de consideração crítica sobre temáticas distintas.

Programa arquitetônico é a descrição do problema arquitetônico a ser abordado durante o projeto (Cherry, 1999; Peña \& Parshall, 2001). A definição e quantificação espacial é o limite usual do desenvolvimento destes trabalhos. No entanto, a descrição do desempenho esperado dos distintos sistemas de um edifício é vista como parte do programa arquitetônico por diversos autores (Cherry, 1999; Peña \& Parshall, 2001; Sanoff, 1977).

Projeto baseado em evidências (Evidence Based Design) consiste no uso de conhecimento científico para amparar a tomada de decisões no projeto arquitetônico (Hamilton \& Watkins, 2009). A aplicação de "selos verdes", parte do principio de que existem parâmetros desejáveis de desempenho que podem e devem ser comprovados de forma qualitativa e quantitativa. A premissa de Hamilton (2009), que projetos sustentáveis devem ser fundamentados em conhecimento e evidência foi adotada como base para este estudo.

Outro conceito importante é o de nível de detalhamento de modelos BIM necessário ás diferentes etapas de desenvolvimento de projeto. A falta deste entendimento é reconhecida como um dos obstáculos para a implementação bem sucedida de tecnologias BIM (Clayton et al., 2008). Para efeitos deste estudo são considerados os seguintes níveis de detalhamento (LOD) de modelos BIM proposto pelo Instituto Americano de Arquitetos:
LOD 100 - Elemento de modelo na forma de narrativa com foco programático, sem necessariamente chegar a uma representação gráfica.

LOD 200 - Elemento de modelo já graficamente representado de forma genérica com dimensões, quantidades, forma, localização e orientação aproximadas.

LOD 300 - Elemento de modelo graficamente representativo de um sistema, objeto ou montagem específica. Neste nível o elemento de modelo é descrito graficamente em termos de dimensões, quantidades, forma, localização e orientação.

\section{Método: 0 Estudo de Caso}

$\mathrm{O}$ estudo de caso foi dividido em dois momentos. O primeiro revisa a literatura a respeito de produtos gráficos e tabulares, sobre sustentabilidade e eficiência energética que podem ser obtidos a partir de modelos BIM para uso no experimento. 0 critério de seleção foi seu uso em analises de edificações de baixa complexidade e aplicabilidade a distintas etapas do processo de projeto. Foram consideradas as etapas de partido, estudo preliminar e anteprojeto. Para cada etapa de projeto foi adotado um nível de detalhamento de modelagem (LOD) específico.

No segundo momento, foi desenvolvido experimentalmente um anteprojeto de residência unifamiliar em João Pessoa-PB/Brasil com programa arquitetônico previamente definido, valendo-se das ferramentas selecionadas. Os métodos para o desenvolvimento do projeto seguiram os princípios de reflexão na ação descritos por Donald Schon (1987). Foram coletados dados sobre o tempo total de desenvolvimento, tempo de modelagem, tempo para obtenção de produtos, tempo de consumo dos produtos, quantidade de transformações, quantidade de avaliações e iterações de projeto. $O$ experimento serve como mecanismo de validação do processo proposto.

O objetivo do estudo foi à identificação de diferentes estágios de modelagem compatíveis com questões específicas de sustentabilidade relevantes para o desenvolvimento de um projeto arquitetônico. Três objetivos específicos nortearam o trabalho:

- caracterizar produtos de diferentes complexidades alcançáveis de forma prática a partir de modelos BIM integrados a ferramentas como Design Builder, Vasari, Solar Tool, Ecotect, e Navisworks.

- caracterizar mecanismos de consumo destes produtos, de maneira integrada ao processo projetual.

- caracterizar o processo de projeto, modelagem e simulação do desempenho da edificação de forma a integrar questões a respeito de sustentabilidade.

Para a definição do processo foram incorporados 4 ciclos de proposição análise e síntese ao processo de projeto. Cada ciclo vincula-se a produtos que podem ser extraídos a partir das 


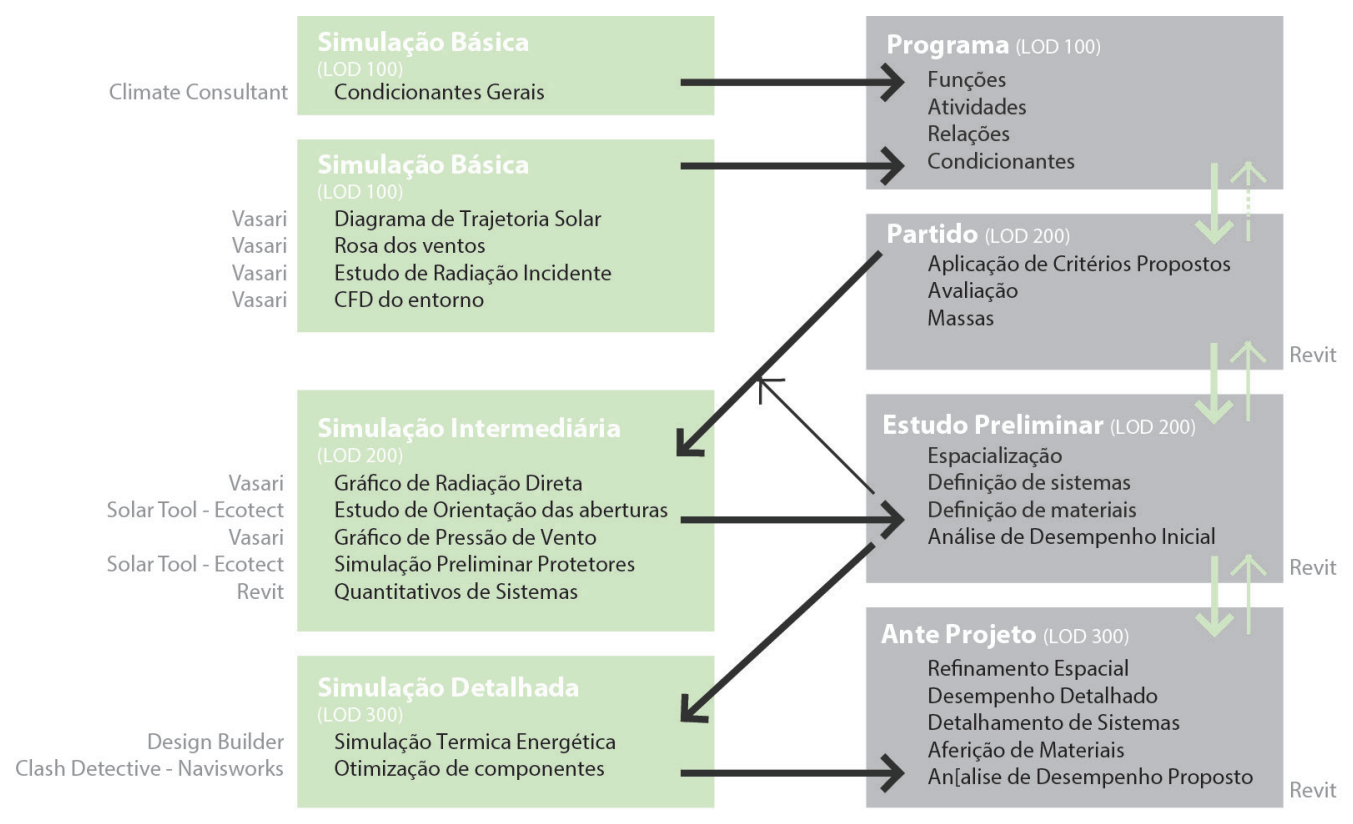

Figura 1: Processo de integração proposto.

ferramentas selecionadas. A figura a seguir descreve os produtos selecionados, mecanismos de analise, indicações de aplicações e forma de consumo no projeto.

\section{Resultados}

As ferramentas escolhidas para o experimento são divididas em ferramentas de análise climática, solar, de simulação térmicas e energéticas e simulação de CFD. Dentro de uma perspectiva de integração de múltiplos produtos foram adotadas ferramentas interoperáveis com o REVIT 2014.

\section{Uso de ferramentas de análise climática (LOD 100)}

A primeira etapa foi a compreensão do macro clima e estabelecimento de estratégias bioclimáticas e eficientes. Foi utilizado o Climate Consultant 5.4 para gerar gráficos e recomendações projetuais a partir de um ano climático de referência.

João Pessoa, localizada no litoral do Nordeste brasileiro, latitude $07^{\circ} 06^{\prime} 54^{\prime \prime} \mathrm{S}$ e longitude $34^{\circ} 51^{\prime} 47^{\prime \prime} \mathrm{O}$, possui clima quente e úmido. Apresenta duas estações bem definidas: verão e época de chuvas no período de janeiro a agosto. A temperatura ao longo do dia varia entre 24 a 31C. A análise bioclimática indica uma predominância de desconforto por calor. O percentual das horas de desconforto por frio é inexpressivo.

A ventilação natural é a principal estratégia projetual, sendo relevante todo ano. Em segundo lugar está o sombreamento de aberturas. A radiação solar é alta principalmente entre as 10 e 16 horas. Ganhos térmicos por radiação devem ser evitados nos ambientes através de sombreamento e uso de cores claras.
Os estudos de modelagem de entorno foram realizados no Autodesk Vasari. Localizado o terreno, o usuário automaticamente insere um arquivo-climático, os dados de entrada são configurados pelo software através de um banco de dados on-line. A modelagem do entorno imediato deve ser realizada, pois contribui na avaliação do impacto da vizinhança no futuro edifício, ou vice e versa. Os produtos extraídos foram:

A análise da orientação do terreno e trajetória solar, para aprimorar a compreensão sobre a orientação, forma mais eficiente do partido arquitetônico e também repercussões sobre elementos de sombreamento. A análise de incidência de radiação na superfície do terreno. Permite verificar se o entorno provoca sombreamento significativo no terreno e também quais áreas são as mais sombreadas. Esta etapa colabora com o zoneamento e definição de prescrições paisagísticas. Neste exemplo foi realizado simulação para o ano todo.

A geração da rosa dos ventos de João Pessoa permitiu a compreensão da direção e frequência dos ventos. Assim, o usuário identifica onde posicionar aberturas de entrada e saída de ar, ou identifica ventos indesejáveis. No exemplo, utilizamos a rosa dos ventos para o ano todo. O regime de ventos em João pessoa a ventilação é abundante em todo quadrante que varia entre leste e sul.

A simulação no túnel de vento(CFD). Colabora com o entendimento da influência do entorno na ventilação no terreno. A partir dessas informações, é possível estabelecer estratégias de ventilação. Verificou-se que quando a ventilação é predominantemente leste, o terreno está favorecido. Porém quando os ventos sopram na direção predominantemente sul, o 


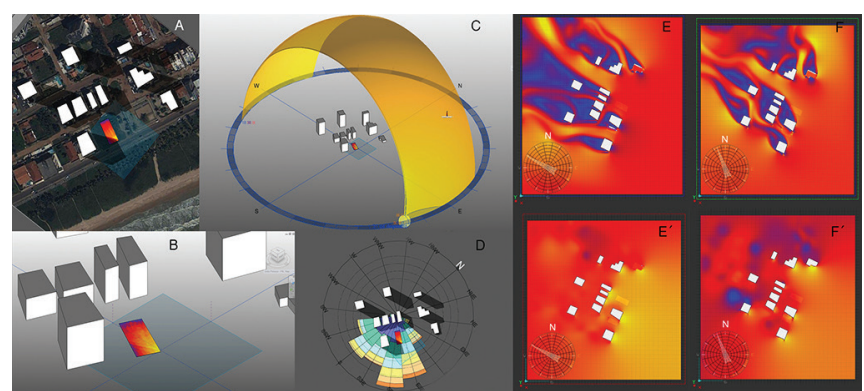

Figure 2: Análises no Vasari. " $A$ " e " $B$ " incidência de radiação solar no terreno; " $C$ " trajetória solar e orientação do terreno; $D$ rosa dos ventos no contexto; "E" e "F" estudos de CFD de velocidade do ar e pressão do vento.

edifício vizinho provoca uma sombra de ventos no terreno, que reduz a pressão do vento na área.

\section{Estudos de massa (LOD 200)}

Os primeiros estudos de massa e partido também foram executados no Autodesk Vasari. A partir de ciclos de iteração de propostas de massa, com análises de ventilação, estudos para otimizar ventilação cruzada e estudos de incidência de radiação nas fachadas, foi estabelecido o partido do projeto arquitetônico. O partido escolhido é composto por dois prismas elevados do solo.

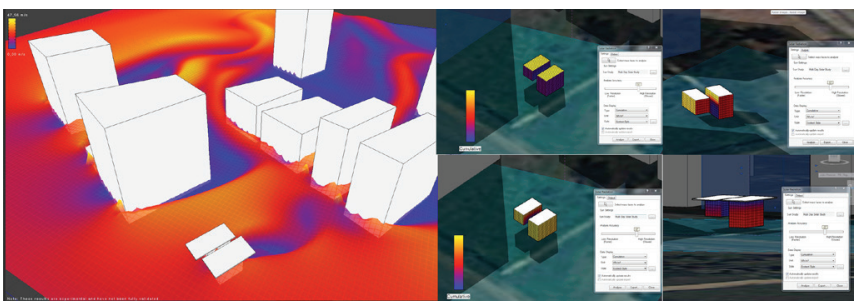

Figura 1: análises do partido arquitetônico.

O partido também levou em consideração questões de segurança, privacidade, iluminação natural e racionalização da construção. Os conceitos que nortearam suas dimensões são: coordenação modular, uso de planta livre, maximização da ventilação cruzada e entrada de luz natural, condensação das áreas molhadas e pavimentos elevados para conservar a permeabilidade do terreno.

Seguiu-se o uso do software Solartool para estabelecer os elementos de sombreamento de superfícies envidraçadas. As máscaras de sombra são geradas automaticamente enquanto se estabelece parâmetros na modelagem. Após o final desse processo, o arquivo originado no Vasari foi migrado para o Revit para dar início ao detalhamento do projeto.

\section{Simulação complexa - Design Builder}

A partir do modelo BIM foi possível extrair o arquivo da simulação complexa, ou seja, não houve uma etapa de modelagem no DesignBuilder. Apenas caracterização e aferição de resultados. Isto possibilitou um grande ganho de tempo se comparado a processos tradicionais. A integração entre o Revit e o DesignBuilder foi a partir do formato GBXML.

O modelo teve o sistema construtivo detalhado e simplificações geométricas devido às limitações do software. Adotou-se uma rotina comum para o modelo com tempo de ocupação de 24 horas por dia. Todos os ambientes foram simulados sem condicionamento artificial, e o número de trocas de ar horárias por ventilação natural foi calculada pelo software.

Para análise dos resultados, foram consideradas a média das temperaturas operativas internas horárias para cada mês que foram comparados às temperaturas da zona de conforto para condições sem movimento de ar e com movimento de ar. Também analisou-se o comportamento térmico dos diferentes elementos do projeto.

Verificou-se que, apesar do sucesso das estratégias bioclimáticas se faz necessário o uso de isolamento no piso. A simulação mostrou que há ganho térmico através do piso que prejudica o desempenho final. Neste caso o contato do piso inferior com o solo promove perda de calor do ambiente interno. Como o projeto prevê o uso de pilotis e palafita, corrigiu-se o problema com uso de isolante térmico. $\mathrm{Na}$ etapa seguinte foi realizada outra simulação que foi considerada satisfatória com dados de temperatura dentro da zona de conforto.

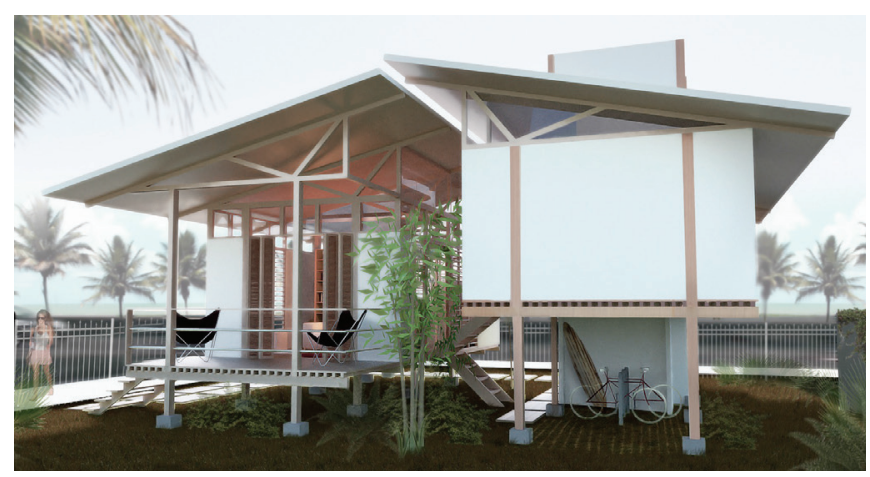

Figura 4: Imagem ilustrativa representando edifício residencial com soluções estabelecidas no anteprojeto. Autor: Natalia Queiroz.

\section{Compatibilização de sistemas (LOD 300)}

A compatibilização do sistema estrutural foi realizada no NavisWork 2014 por meio da ferramenta Clash Detective. Foi extraído um relatório de conflito entre peças estruturais no projeto que apontou dez conflitos entre elementos estruturais. A partir do relatório foram identificados os motivos dos conflitos. A otimização do sistema deu-se por meio da adoção de um detalhamento de encaixe estrutural. Por se tratar de ferramentas interoperáveis, o processo consistiu apenas da importação do modelo para a ferramenta de compatibilização de sistemas e posterior atualização do relatório. (Figura 5). 


\section{Discussão de Resultados}

O tempo total de modelagem alcançar o anteprojeto apresentado foi de 46,3 horas. Estima-se que para o mesmo anteprojeto, um processo tradicional apoiado em modelos CAD sem o uso de simuladores básicos duraria pelo menos 112 horas, sem considerar conflitos e retrabalho oriundos de erros de projeto. Caso o modelo tradicional utilizasse ferramentas complexas para as simulações apresentadas, o tempo estimado sobe para 161,6 horas (Figura 6).

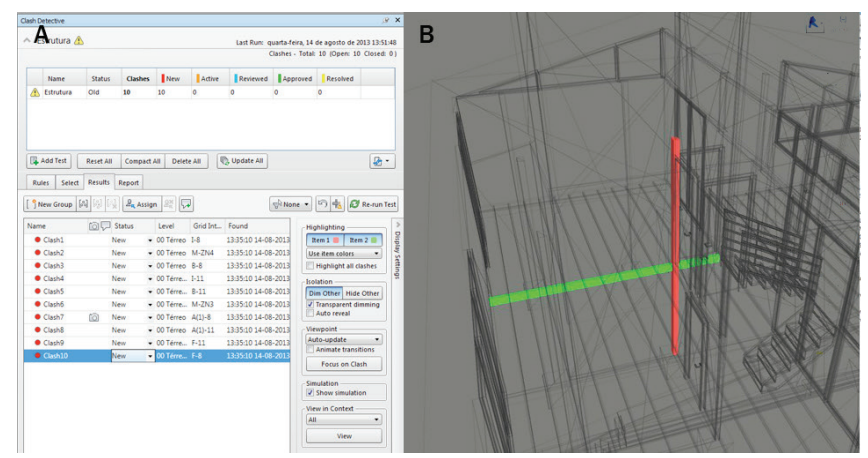

Figura 5: Detecção e correção de conflitos utilizando Navisworks e Revit 2014.

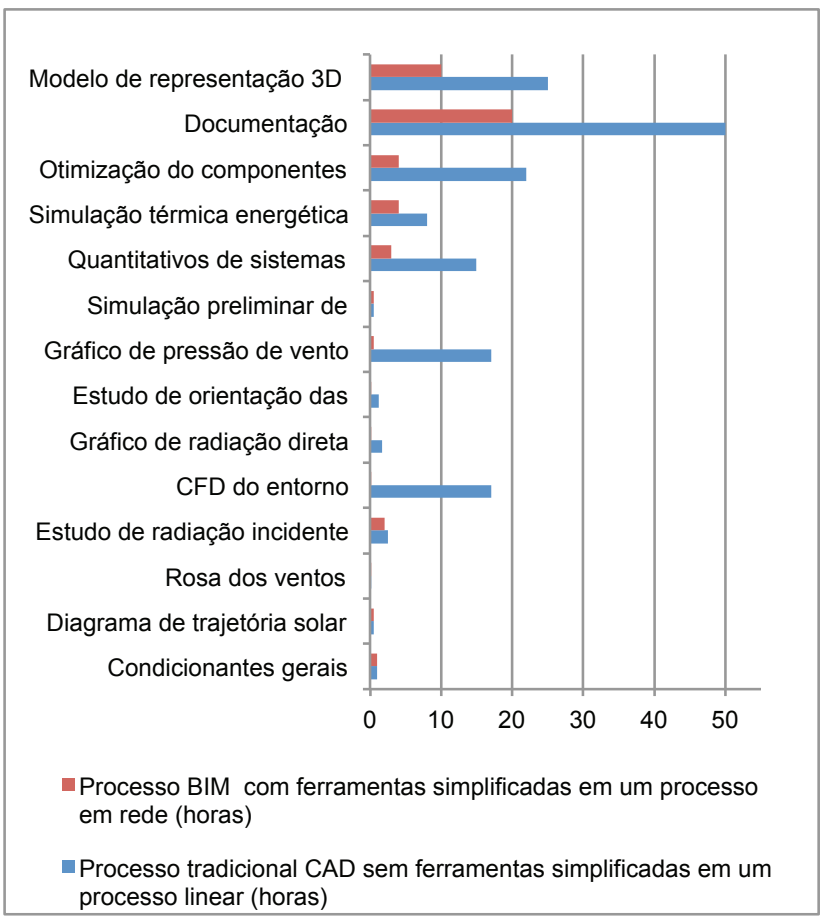

Figura 6: Comparativo de horas do processo proposto e processo tradicional.

\section{Conclusão}

A partir dos produtos selecionados foi possível integrar discussões relativas à sustentabilidade e eficiência energética ao desenvolvimento do anteprojeto. Conforme apresentado, o estudo de caso identifica os diferentes estágios de modelagem compatíveis com questões específicas de sustentabilidade relevantes para o desenvolvimento de um projeto arquitetônico. Verificou-se, através do registro de horas trabalhadas uma redução significativa do tempo de desenvolvimento projetual através do processo proposto, quando comparado a processos tradicionais. Fica evidente para os autores que a proposta coloca a integração destas questões ao alcance de projetistas versados em ferramentas BIM e simulação térmica.

O estudo limita-se a seleção de produtos indicada, a qual reconhecidamente não é exaustiva. Sabe-se também que a utilização de profissionais com conhecimento específico nas ferramentas propostas, bem como na análise dos produtos utilizados, foi fator determinante nos resultados obtidos. No entanto, serve como indicativo válido do potencial de processos integrados de projeto. A adaptação do estudo de caso para uso em meios acadêmicos servirá como suporte para o desenvolvimento de componentes curriculares focados em projetos integrados.

\section{Referências}

AIA-CC. (2007). Integrated Project Delivery: A Guide AIA-CaliforniaCouncil (Ed.)

Bachtold, F. (2008). Resíduos da construção civil supera o lixo doméstico em capitais. http://www1.folha.uol.com.br/folha/cotidiano/ult95u429454.shtml

Boddy, S., Rezgul, Y., Cooper, G., \& Wetherill, M. (2007). Computer integrated construction: A review and proposals for future direction. Advances in Engineering Software, 38(10), 677-687. doi: 10.1016/j.advensoft.2006.10.007

Brasil, A. A. n. d. e. e. (2002). Atlas de energia elétrica do Brasil. Brasília: Aneel.

Cherry, E. (1999). Programming for design : from theory to practice. New York: John Wiley.

Clayton, M. J., Johnson, R. E., Vanegas, J., Nome, C. A., Ozener, O. O., \& Culp, C. E. (2008). Downstream of Design: Lifespan Costs and Benefits of Building Information Modeling. College Station: Texas A\&M University.

Eastman, C. M. (2008). BIM handbook : a guide to building information modeling for owners, managers, designers, engineers, and contractors. Hoboken, N.J.: Wiley.

Friedrich -Naumann-Stiftung. (1988). An Agenda for our common future : implications of the Bundtland Commission report. New York, N.Y.: Friedrich Naumann Foundation.

Green building rating system : for new construction and core \& shell projects : based on LEED 2009, reference guide. (2011). Hyderabad: Indian Green Building Council, Confederation of Indian Industry.

Hamilton, D. K., \& Watkins, D. H. (2009). Evidence-based design for multiple building types. Hoboken, N.J.: John Wiley \& Sons, Inc.

Lamberts, R., L. Dutra, et al. (2004). Eficiência Energética na Arquitetura. São Paulo: ProLivros.

Mendes, N., Westphal, F. S., Lamberts, R., \& Neto, J. A. B. d. C. (2005). Uso de instrumentos computacionais para análise do desempenho térmico e energético de edificações no Brasil. Paper presented at the Ambiente Construido, Porto Alegre.

Peña, W., \& Parshall, S. (2001). Problem seeking : an architectural programming primer (4th ed.). New York: Wiley.

Roaf, S. (2012). Ecohouse : a design guide (Fourth edition. ed.). New York: Architectural Press.

Sanoff, H. (1977). Methods of architectural programming. Stroudsburg, Pa.: Dowden, Hutchinson \& Ross

Schön, D. A. (1987). Educating the reflective practitioner : toward a new design for teaching and learning in the professions (1st ed.). San Francisco: Jossey-Bass.

Venâncio, R. (2012). Modos projetuais de simulação: Uso de ferramentas de simulação térmica no processo projetual de arquitetura. (Doutorado), UFRN, Natal. 